Unfallchirurg $2022 \cdot 125: 323-326$

https://doi.org/10.1007/s00113-021-01025-3

Angenommen: 6. Mai 2021

Online publiziert: 16. Juni 2021

๑ Der/die Autor(en) 2021

\section{Redaktion}

W. Mutschler, München

H. Polzer, München

B. Ockert, München

\section{Anamnese}

Eine 36-jährige angeschnallte Pkw-Beifahrerin auf dem rechten Rücksitz verunfallte im August 2019 (Differenzgeschwindigkeit Delta v $70 \mathrm{~km} / \mathrm{h}$ ) mit Spiralfraktur des Humerus rechts in der 19. Schwangerschaftswoche. Seinerzeit konservative Therapie mit gipsverstärktem Gilchrist.

\section{Befund}

Bei fortdauernden Schmerzen und Funktionseinschränkung im Alltäglichen am 18.02.2020 Oberarm-CT mit Nachweis einer Humeruspseudarthrose (6 Monate nach Unfall) mit um Schaftbreite versetztem Verlauf.

\section{Diagnose}

Humeruspseudarthrose (• Abb. 1).

\section{Therapie}

Am 18.03.2020 Beginn der apparativem Knochenstimulationstherapie als neuartige Kombination als individueller Heilversuch im Einverständnis mit der Patientin aus:

1. hochenergetischer fokussierter extrakorporaler Stoßwellentherapie (ESWT, Storz Ultra mit einem elektromagnetischen Generator $0,3 \mathrm{~mJ} / \mathrm{mm}^{2}, 3000$ Impulse) gemäß den evidenzbasierten Leitlinien u. a. der DIGEST (www.digest-ev.de),

2. extrakorporale Magnetotransduktionstherapie (EMTT, Storz Magne-

Karsten Knobloch (D)

SportPraxis Prof. Knobloch, Hannover, Deutschland

\title{
Knochenstimulation 4.0 - Kombination aus EMTT und ESWT bei Humeruspseudarthrose
}

\section{Ein Fallbericht}

tolith, Energiestufe 8/8, $8 \mathrm{~Hz}, 6000$ Impulse/Sitzung). Die EMTT ist eine Weiterentwicklung der bekannten „pulsed electromagnetic field therapy (PEMF)“, für die positive Effekte auf die Knochenheilung [1-3] wie auch für die Osteointegration von Implantaten $[4,5]$ beschrieben sind beide benutzen oszillierende Magnetfelder. Jedoch unterscheiden sich EMTT-Geräte von den PEMF-Geräten in 2 wesentlichen physikalischen Parametern:

1. Die Oszillationsfrequenz ist beim EMTT mit $7 \mu$ s vs. $280 \mu$ s beim PEMF ungleich schneller und hochfrequenter. Damit Magnetfelder eine biologische Wirkung erzielen, ist die Überwindung einer „Schwellendosis“ von $10 \mathrm{mT}$ nötig: Ein einzelner Impuls eines EMTT-Gerätes erreicht dies 10mal in $70 \mu \mathrm{s}$, ein PEMF-Gerät genau einmal in $280 \mu$ s.

2. Die elektromagnetische Transduktionsleistung, gemessen in $\mathrm{kT} / \mathrm{s}$, ist bei PEMF-Geräten definitionsgemäß $<60 \mathrm{kT} / \mathrm{s}$, bei EMTT-Geräten $>60 \mathrm{kT} / \mathrm{s}$ - sprich die Leistung der EMTT-Geräte ist ungleich höher im Vergleich zu PEMF-Geräten.

Diese Kombination aus fokussierter ESWT und EMTT wurde 3-malig im Abstand von einer Woche im März/ April 2020 während des ersten Coronalockdowns ambulant durchgeführt, mit 95,9 J Gesamtenergie. Am 06.05.2020 Humerus-Dünnschichtbilddiagnostik als digitale Volumentomographie (SCS
MedSeries H22). Hier erfreulicherweise keine Frakturlinie der Pseudarthrose mehr nachweisbar, sprich Konsolidierung der Humeruspseudarthrose bereits 5 Wochen nach der letzten Knochenstimulationsbehandlung. Die Funktion des Armes konnte daraufhin im Alltag wiederhergestellt werden, mit einem DASH-Score von 12 Punkten im Juli 2020, acht Wochen nach der Bildgebung (von 63 Punkten kommend im März).

\section{Fallanalyse}

Pseudarthrosen des Humerus treten in bis $\mathrm{zu} 15 \%$ der Humerusfrakturen auf [6]. Jüngst wurden in dieser Zeitschrift die konservativen Therapieoptionen von Pseudarthrosen von Großner und Schmidmaier lesenswert beleuchtet [7]. Der Einsatz der fokussierten Stoßwellentherapie (ESWT) zur Behandlung von Pseudarthrosen der langen Röhrenknochen ist seit den frühen 1990erJahren u. a. durch die Habilitationsschrift von Prof. Ekkernkamp [8] in Bochum und weiteren intensiv studiert und beschrieben [9].

Lokalisationsabhängig wurden aber deutliche Unterschiede der Erfolgsraten berichtet. So erreichte man eine knöcherne Konsolidierung bei $\mathrm{Hu}$ meruspseudarthrosen mit fokussierter hochenergetischer ESWT mit dem Ossatron von HMT (2500-4000 Impulse, $0,25-0,4 \mathrm{~mJ} / \mathrm{mm}^{2}$ ) anfangs bei nur einem von 5 behandelten Patienten [10] - sprich in $20 \%$. In einer Kohorte mit $13 \mathrm{Hu}$ meruspseudarthrosen [11] im Jahr 2009 wurde die hochenergetische fokussierte 

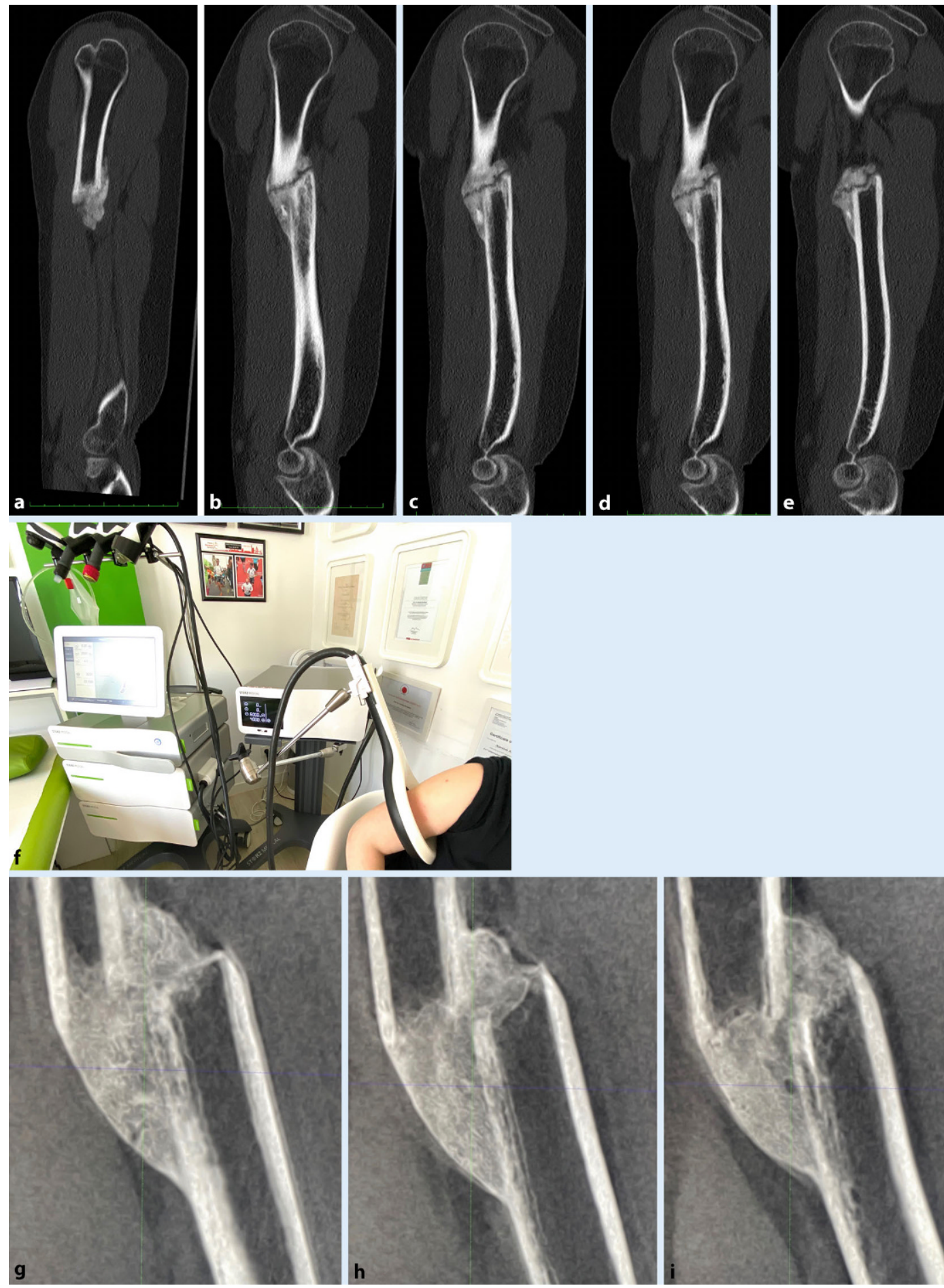

Abb. $1<$ a-e OberarmCT mit Humeruspseudarthrose 6 Monate nach Verkehrsunfall. f Extrakorporale Magnetotransduktionstherapie (EMTT) mit Storz Magnetolith ${ }^{\circledR}$ (Storz Medical, Tägerwilen, Schweiz) zur Knochenstimulationstherapie in Kombination mit der fokussierten extrakorporalen Stoßwellentherapie (ESWT). g-i Digitale Volumentomographie des Humerus 5 Wochen nach 3 Knochenstimulationssitzungen mit vollständiger Konsolidierung der Humeruspseudarthrose

Stoßwellentherapie mit 4000 Impulsen bei etwas höherer Energieflussdichte von $0,56 \mathrm{~mJ} / \mathrm{mm}^{2}$ mit dem vorgenannten Ossatron von HMT (Schweiz) angewendet, mit einer Therapiesitzung in örtlicher Betäubung. Die Heilungsraten der Humeruspseudarthrosen waren zeitabhängig 2 Monate nach ESWT $8 \%$ (1/13), 3 Monate nach ESWT bei $23 \%$
(3/13) und 6 Monate nach ESWT bei $62 \%(8 / 13)$, mithin offenbar besser als die eingangs berichteten $20 \%$ Erfolg.

In dieser Zeitschrift berichtet die unfallchirurgische Klinik der Medizinischen Hochschule Hannover (MHH) im Jahr 1999 [12] von 27 Pseudarthrosen an langen Röhrenknochen (11 bereits mit Reosteosynthesen), mit einer Er- folgsrate von $41 \%$ nach 6 Monaten durch die 2-malige fokussierte ESWT $\left(0,2 \mathrm{~mJ} / \mathrm{mm}^{2}\right)$ an langen Röhrenknochen. Von den 2 eingeschlossenen Oberarmpseudarthrosen kam es nur in einem Fall (metaphysär, 24 Jahre alt, ESWT 28 Wochen nach Operation) zur Ausheilung, sprich 50 \%iger Erfolgsrate am Humerus. 
vs. EMTT

Effektive Transduktionsleistung (kT/s)
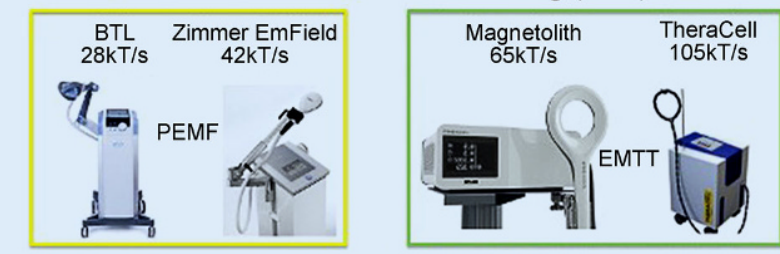

Die Ergänzung der EMTT über oszillierende Magnetfelder zeigt in diesem Fall eine rasche Konsolidierung der Humerusschaftpseudarthrose. Bei Pseudarthrosen des Kahnbeins und der Mittelhand ist der kombinierte Einsatz der fokussierten ESWT und EMTT jüngst beschrieben $[13,14]$. Im Unterschied zur "pulsed magnetic field therapy (PEMF)“ mit einer effektiven Transduktionsleistung $<60 \mathrm{mT} / \mathrm{s}$ zeichnen sich EMTT-Geräte wie das Storz Magnetolith $^{\circledR}$ durch eine effektive Transduktionsleistung $>60 \mathrm{kT} / \mathrm{s}$ und eine 40 fach höhere Oszillationsfrequenz im Vergleich zur PEMF aus - sprich, es bestehen physikalische Unterschiede in der biophysikalischen Gerätetechnik (• Abb. 2).

Der weitere Einsatz der EMTT in Ergänzung oder auch allein zur Knochenstimulationstherapie ist in prospektiven Studien zu prüfen.

\section{Fazit für die Praxis}

\section{- Die Kombination der fokussierten \\ ESWT und der extrakorporalen Magnetotransduktionstherapie (EMTT) scheint die Knochenheilung bei Humeruspseudarthrosen rasch zu stimulieren. \\ - Es handelt sich um eine nichtinvasive, ambulant und schmerzfrei durchführ- bare Therapie, auch bei einliegenden „Magnet(MR)-sicheren“ Implantaten, ohne Nebenwirkungen.}

\section{Korrespondenzadresse}

Prof. Dr. Karsten Knobloch, FACS

SportPraxis Prof. Knobloch

Heiligerstr. 3, 30159 Hannover, Deutschland professor.knobloch@sportpraxis-knobloch.de
Abb. $2<$ Effektive Transduktionsleistung von „Pulsedelectromagnetic-field(PEMF)"Geräten $<60 \mathrm{kT} / \mathrm{s}$ und "Extracorporeal-magnetotransductiontherapy(EMTT)"Geräten $>60 \mathrm{kT} / \mathrm{s}$

\section{Einhaltung ethischer Richtlinien}

Interessenkonflikt. K. Knobloch ist amtierender Präsident der deutschsprachigen int. Gesellschaft für Stosswellentherapie DIGEST und Vorstandsmitglied der DIGEMTT. Er hat Vorträge gehalten für Canon Medical, TRB Chemedica, Storz Medical, Medi. In Bezug auf diese Fallvorstellung liegen keine weiteren Interessenkonflikte vor.

Für diesen Beitrag wurden vom Autor keine Studien an Menschen oder Tieren durchgeführt. Für die aufgeführten Studien gelten die jeweils dort angegebenen ethischen Richtlinien. Für Bildmaterial oder anderweitige Angaben innerhalb des Manuskripts, über die Patienten zu identifizieren sind, liegt von ihnen und/ oder ihren gesetzlichen Vertretern eine schriftliche Einwilligung vor.

Open Access. Dieser Artikel wird unter der Creative Commons Namensnennung 4.0 International Lizenz veröffentlicht, welche die Nutzung, Vervielfältigung, Bearbeitung, Verbreitung und Wiedergabe in jeglichem Medium und Format erlaubt, sofern Sie den/die ursprünglichen Autor(en) und die Quelle ordnungsgemäßnennen, einen Link zur Creative Commons Lizenz beifügen und angeben, ob Änderungen vorgenommen wurden.

Die in diesem Artikel enthaltenen Bilder und sonstiges Drittmaterial unterliegen ebenfalls der genannten Creative Commons Lizenz, sofern sich aus der Abbildungslegende nichts anderes ergibt. Sofern das betreffende Material nicht unter der genannten Creative Commons Lizenz steht und die betreffende Handlung nicht nach gesetzlichen Vorschriften erlaubt ist, ist für die oben aufgeführten Weiterverwendungen des Materials die Einwilligung des jeweiligen Rechteinhabers einzuholen.

Weitere Details zur Lizenz entnehmen Sie bitte der Lizenzinformation auf http://creativecommons.org/ licenses/by/4.0/deed.de.

\section{Literatur}

1. Varani K, Vincenzi F, Pasquini S, Blo I, Salati S, Cadossi M, De Mattei M (2021) Pulsed electromagnetic field stimulation in osteogenesis and chondrogenesis: signaling pathways and therapeutic implications. Int J Mol Sci 22(2):809

2. Liu Y, Hao L, Jiang L, Haitao L (2021) Therapeutic effect of pulsed electromagnetic field on bone wound healing in rats. Electromagn Biol Med 40(1):26-32
3. Habib M, Horne DA, Hussein K, Coughlin D, Waldorff El, Zhang N, Ryaby J, Lotz JC (2020) Magnetic nanoparticles synergize with pulsed magnetic fields to stimulate osteogenesis in vitro. Tissue Eng Part A. https://doi.org/10.1089/ten. TEA.2020.0102

4. Nayak BP, Dolkart O, Satwalekar P, Kumar YP, Chandrasekar A, Fromovich O, Yakobson E, BarakS Dayube U, Shibli JA (2020) Effect of pulsed electromagnetic field (PEMF) on dental implants stability: a randomized controlled clinical trial. Materials 13(7):1667

5. Cai J, Shao X, Yang Q, Yang Y, Yan Z, Luo E, Feng $X$, Jing D (2020) Pulsed electromagnetic fields modify the adverse effects of glucocorticoids on bone architecture, bone strength and porous implant osseointegration by rescuing boneanablicactions. Bone 133:115266

6. Leiblein M, Verboket R, Marzi I, Wagner N, Nau C (2019) Nonunions of the humerus. Chin JTraumatol 22:187-195

7. Großner T, Schmidmaier G (2020) Konservative Therapieoptionen der Pseudarthrosen. Unfallchirurg 123:705-710

8. Ekkernkamp A (1991) Die Wirkung extrakorporaler Stosswellen auf die Frakturheilung - eine tierexperimentelle Studie. Habilitationsschrift an den Berufsgenossenschaftlichen Krankenanstalten „Bergmannsheil', Ruhr-Universität Bochum

9. Haupt G, Haupt A, Ekkerkamp A, Gerety B, Chvapil M (1992) Influence of shock waves on fracture healing. Urology 39(6):529-532

10. Schaden W (1997) Clinical experience with shock wave therapy of pseudarthrosis, delayed fracture healing, and cement-free endoprosthesis loosening. In: Siebert W, Buch M (Hrsg) Extracorporeal shock waves in orthopedics. Springer, Berlin, Heidelberg

11. Xu ZH, Jiang Q, Chen DY, Xiong J, Shi DQ, Yuan T, Zhu XL (2009) Extracorporeal shockwave treatment in nonunions of long bone fractures. Int Orthop 33:789-793

12. Beutler S, Regel G, Pape HC, Machtens S, Weinberg AM, Kremeike I, Jonas U, Tscherne H (1999) Extracorporeal shock wave therapy for delayed union of long bone fractures-preliminary results of a prospective study. Unfallchirurg 102(11):839-847

13. Knobloch K (2021) Extracorporeal magnetotransduction therapy (EMTT) \& high-energetic focused ESWT as bone stimulation therapy for metacarpal nonunion. Handchir Mikrochir Plast Chir 53(1):82-6. https://doi.org/10.1055/a-1344-8126

14. Knobloch K (2020) Novel extracorporeal magnetotransduction therapy with Magnetolith and highenergy focused electromagnetic extracorporeal shockwave therapy as bone stimulation therapy for scaphoid nonunion-a case report. Med Case RepStudyProtoc 2(1):e0028 
Hier steht eine Anzeige.

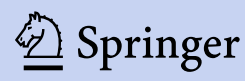

